\title{
THE LIMITATIONS OF CANINE TRABECULAR BONE AS A MODEL FOR HUMAN: A BIOMECHANICAL STUDY
}

\begin{abstract}
J. L. Kuhn, S. A. Goldostein, M. J. Ciarelli and L. S. Matthews
The Biomechanics, Trauma, and Sports Medicine Laboratory, The University of Michigan, Section of Orthopaedics, Department of Surgery, and the Bioengineering Program, Ann Arbor, MI. U.S.A.

Abstract--Distal canine femurs were sectioned into $8 \mathrm{~mm}$ cubic specimens. Orthogonal compression tests were performed to preyield in two or three directions and to failure in a third. Apparent density and ash weight density were measured for a subset of specimens. The results were compared to the human distal femur results of Ciarelli et al. (Transactions of the 32nd Annual Meeting of the Orthopaedic Research Society, Vol. 11, p. 42, 1986). Quantitative similarities existed in the fraction of components comprising the trabecular tissue of the two species. Qualitative similarities were seen in the positional and anisotropic variation of the mechanical properties, and also in the form and strength of the relationships between the mean modulus and bone density, ultimate stress and density, and ultimate stress and modulus. However. significantly different regression equations resulted for the mean modulus-density, and ultimate stress-modulus relationships, indicating that for the same density, canine trabecular bone displays a lower modulus than human, and may achieve greater compressive strains before failure
\end{abstract}

\section{INTRODUCTION}

Canine bone has served extensively as a model for human bone in experimental musculoskeletal research. Through such research, insight has been gained into a variety of orthopedic problems such as cortical bone transplantation (Bos et al. 1983; Enneking et al., 1975), bone metabolism (Harris et al., 1968, 1972), porous ingrowth (Bobyn et al., 1980; Ducheyne et al., 1977; Galante et al., 1971), and bone remodeling (Burr et al., 1985; Chamay and Tschantz, 1972; Goldstein et al., 1986).

The efficacy of an animal model may be dependent on the systems or processes being investigated and therefore, each specific research protocol must be investigated in order to assess the value of experimental results. For instance, in osteoporosis research, prime considerations in the choice of an animal model are the characteristics of trabecular and cortical bone remodeling, as well as age related changes in bone morphology. Adult dogs have been shown to exhibit analogous remodeling and age related changes associated with osteoporosis in humans (Detenbeck and Jowsey, 1969; Faugere et al., 1985; Jee, 1980; Martin et al., 1981; Snow et al., 1984; Snow and Anderson, 1986), supporting their use as models for studying mechanisms and treatments for this bone disease. Recently, Vahey et al. (1987) published a study on the mechanical properties of canine trabecular bone in the proximal femur. Their results demonstrated a qualitative similarity in properties to human trabecular bone of the same anatomical region, and thus supported the use of canine trabecular bone as a suitable model.

The purpose of this paper is to examine the limitations of the canine distal femur as a model for

Received in final form 9 May 1988. trabecular bone biomechanical studies in humans, by comparing mechanical properties, and relationships between modulus, strength, and density.

\section{MATERIALS AND METHODS}

Twenty-two large, skeletally mature, mongrel dog femurs (nine pairs, four singles) were obtained within $2 \mathrm{~h}$ of death, cleaned of adhering soft tissue, and stored frozen at $-10^{\circ} \mathrm{C}$. To enable a fixed and consistent positioning of all bones for sectioning, the proximal ends of the femurs were embedded in 2 in. square aluminum blocks using a plaster mix (Vel-Mix Stone, Kerr Co., Chicago, Illinois). The aluminum blocks were securely clamped into vice grips on the $x-y$ table of a numerical milling machine (Model Series I, CNC, Bridgeport, Troy, MI). Under constant water irrigation and using a stainless steel blade rotating at low speed, the femoral condyles were removed to expose a first plane of trabecular bone. Eight millimeter bone cubes were cut from the metaphysis with the cube axes corresponding to the anterior-posterior, medial-lateral and inferior-superior anatomical axes. Those bone cubes originating from the most distal $8 \mathrm{~mm}$ layer were designated as specimens from layer 1 . Successive $8 \mathrm{~mm}$ layers in the proximal direction were numbered in increasing order. The actual dimensions of each bone cube were measured with a micrometer, and the distal face was marked so that specimen identification and orientation could be documented throughout the study.

In a manner similar to the testing protocol of Brown and Ferguson (1980), 130 specimens were orthogonally tested in uniaxial compression at a strain rate of $1 \% \mathrm{~s}^{-1}$ on a materials testing machine (Model 1000 Universal, Instron), at room temperature. All specimens were kept moist during testing. In order to 
evaluate the precision of the results from pre-yield compression tests, a subset of 35 specimens was tested to preyield in all three directions, and then to failure in one direction. In this way, the modulus determined in a test to preyield could be compared to the modulus determined from the test to failure in the same direction. The remaining specimens were tested to preyield in two directions and to failure in the third direction. Prior to each compression test, specimens were cyclically preloaded three to six times to approximately $20 \%$ of ultimate stress before actual loading began in that direction. The force-deflection data were acquired directly using an analog to digital converter and a Tektronix 4054 computer, and simultaneously monitored on an oscilloscope (Model 5103N, Tektronix) to prevent loading past yield. Deflection data were based on the cross head motion of the materials testing machine, then corrected for system compliance. The stiffness of the Instron Model 1000 with our testing platen was $10,000 \mathrm{~N} \mathrm{~mm}^{-1}$.

Normalizing by specimen cross-sectional area and height, the digital data were converted to stresses and strains. To calculate the modulus, linear regressions were performed on the stress-strain data contained in the most linear portion of the curve. It should be recognized that these moduli determined in three orthogonal directions were not intended to describe orthotropic material constants, as such a characterization would require investigation of the principal material axes. Ultimate stresses were taken from the points of maximum load.

Apparent density was measured for 41 cubes (originating from the single femurs of four different animals and a pair of femurs from a fifth animal), and ash weight density measured for 37 of the 41 cubes, according to the combined protocols of Arnold (1960), Galante et al. (1970), and Carter and Hayes (1977). Apparent density was defined as wet weight ( $\mathrm{g}$ ) divided by bulk volume $\left(\mathrm{cm}^{3}\right)$, where bulk volume was equal to the product of specimen height, width, and depth as measured with a micrometer. Wet weight was determined by: (1) removing marrow from the specimens with a high pressure stream of tap water, (2) degreasing specimens in ethanol, (3) freeing the excess water by centrifugation (under refrigeration) of the bone cubes for $15 \mathrm{~min}$ at $9000 \mathrm{rpm}$, and (4) weighing the bone specimens. After the apparent density was calculated, the specimens were dried in an oven at $100^{\circ} \mathrm{C}$, then ashed in a furnace at $500^{\circ} \mathrm{C}$ for $60 \mathrm{~h}$. The ash residue was weighed and the ash weight density calculated as ash weight $(\mathrm{g})$ divided by bulk volume $\left(\mathrm{cm}^{3}\right)$.

\section{RESULTS}

Thirty-five compression tests were analyzed for differences between calculation of the modulus in a preyield and failure test in the same direction. The mean percent difference $(\%$ difference $=(1-$ (preyield modulus/failure modulus) $\times 100$ ) in calculated modu- lus for all 35 specimens was $-7.8 \%$. Limits on a $95 \%$ confidence interval for this difference were from $-18 \%$ to $2.7 \%$. It was also observed that this difference in modulus was somewhat dependent on the relative stiffness of the specimen tested. Specimens with preyield moduli greater than $170 \mathrm{MPa}$ had differences centered around $3.6 \% \pm 10.9 \%$, while the large discrepancies occurred only for very compliant trabecular bone.

The mechanical test results showed that the compressive moduli ranged from as low as $5 \mathrm{MPa}$ in the most proximal regions, to $656.9 \mathrm{MPa}$ beneath the articular surface. Because maximal information was desired, cube locations within the cross-sectional geometry of the distal femurs were not standardized nor matched between different femurs. Bone cube locations were divided into four quadrants: posteriormedial, anterior-medial, anterior-lateral, and posterior-lateral. The mean AP, ML, and IS moduli for these quadrants in layers $1-3$ are shown in Fig. 1. Ultimate stresses ranged from 1.33 to $20.76 \mathrm{MPa}$. The mean ash weight was $0.263 \mathrm{~g} \mathrm{~cm}^{-3}$, and the mean apparent density was $0.444 \mathrm{~g} \mathrm{~cm}^{-3}$. In all dog femurs, the greatest stiffnesses were documented in the AP and IS directions, in the posterior condyles and beneath the patellar groove. Lower moduli were displayed by the trabecular bone in the central metaphyseal regions. A noticeable variation through the depth of the metaphyses was observed as modulus values dropped progressively in the proximal direction.

Canine relationships between modulus and ultimate stress with apparent density and ash weight density

Regression models which describe the empirical relationship between the modulus in a preyield test for each test direction and apparent and ash weight density are contained in Table 1 . Both linear regressions and $\log -\log$ regressions showed that approximately $40-60 \%$ of the variance in the modulus data could be explained by differences in density. The $\log$-log regressions indicated relationships of modulus with density raised to between the 1.03 and 1.78 power. The strength of the relationships between preyield moduli and ash weight density were very close to those obtained from moduli-apparent density relationships. As ash weight density and apparent density were almost perfectly correlated $(r=0.99)$, this was not a surprising result.

In order to estimate an overall stiffness for each cube, a mean modulus for each bone cube was calculated from the three moduli in each test direction. Regression equations for mean modulus and apparent and ash weight density are also shown in Table 1 . The mean modulus correlated to density better than any single direction modulus. Approximately $73 \%$ of the variance could be explained by linear regression models, and about $79 \%$ by $\log$-log regression models.

The relationships between ultimate stress and apparent and ash weight density for each direction are 


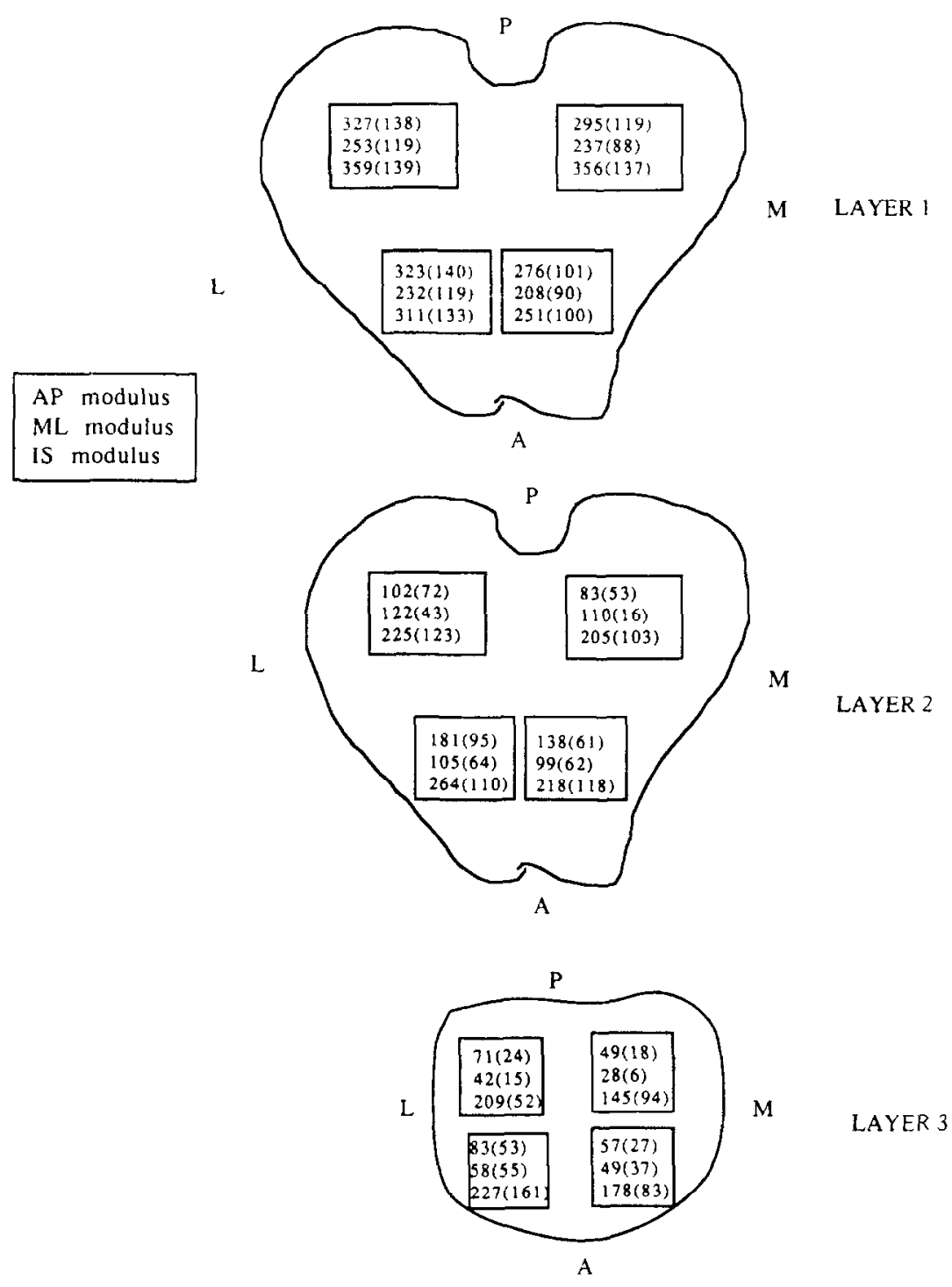

Fig. 1. The mean modulus values in the AP, ML, and IS directions for four quadrants of the distal femoral metaphyseal cross section are shown for three $8 \mathrm{~mm}$ layers. Standard deviations are shown in parentheses.

All values are presented in units of $\mathrm{MPa}$.

Table 1. Linear and $\log -\log$ regression models to express the relationships between modulus in a preyield test and apparent and ash weight density. These models take the form: $E=A x+B$, and $E=C x^{4}$, were $E=$ modulus in the AP, ML, or IS direction in units of $\mathrm{MPa}$, and $x=$ apparent or ash weight density in units of $\mathrm{g} \mathrm{cm}^{-3} \cdot n=41$ for apparent density regressions, $n=37$ for ash weight density regressions

\begin{tabular}{|c|c|c|c|c|c|c|c|c|c|c|c|c|}
\hline & \multicolumn{6}{|c|}{$x=$ apparent density } & \multicolumn{6}{|c|}{$x=$ ash weight density } \\
\hline & $A$ & $B$ & $r^{2}$ & $C$ & $d$ & $r^{2}$ & $A$ & $B$ & $r^{2}$ & C & $d$ & $r^{2}$ \\
\hline$E_{A P}$ & 585.9 & -63.4 & 0.53 & 639.1 & 1.66 & 0.54 & 1043.8 & -73.8 & 0.50 & 1510.2 & 1.62 & 0.52 \\
\hline$E_{M L}$ & 544.0 & -102.0 & 0.58 & 361.4 & 1.59 & 0.57 & 1071.0 & -136.7 & 0.63 & 1118.8 & 1.78 & 0.58 \\
\hline$E_{I S}$ & 511.5 & 21.6 & 0.47 & 542.4 & 1.03 & 0.49 & 859.5 & 22.2 & 0.40 & 962.9 & 1.07 & 0.42 \\
\hline$E_{\text {mean }}$ & 547.1 & -47.9 & 0.73 & 572.5 & 1.39 & 0.79 & 991.4 & -62.8 & 0.72 & 1352.9 & 1.48 & 0.78 \\
\hline
\end{tabular}

listed in Table 2. Also shown is the relationship found when all directions were combined. Both linear and log-log regressions described the data quite well, with squared correlation coefficients ranging from 0.61 to 0.93 .
Table 3 shows the strong linear relationships found between ultimate stress and modulus in compression to failure tests. When all directions were combined, a linear relationship with a squared correlation coefficient of 0.83 was obtained. 
Table 2. Linear and $\log -\log$ regression models to express relationships between ultimate stress in the AP, ML, and IS directions, and apparent and ash weight density. These models take the form: $\sigma=A x+B$, and $\sigma=C x^{d}$, where $\sigma=$ ultimate stress in units of $\mathrm{MPa}$, and $x=$ apparent density or ash weight density in units of $\mathrm{g} \mathrm{cm}^{-3}$

\begin{tabular}{|c|c|c|c|c|c|c|c|c|c|c|c|c|c|c|}
\hline & \multicolumn{7}{|c|}{$x=$ apparent density } & \multicolumn{7}{|c|}{$x=$ ash weight density } \\
\hline & $n$ & $A$ & $B$ & $r^{2}$ & $C$ & $d$ & $r^{2}$ & $n$ & $A$ & $B$ & $r^{2}$ & C & $d$ & $r^{2}$ \\
\hline$\sigma_{A P}$ & 8 & 24.95 & -3.7 & 0.86 & 27.07 & 1.71 & 0.91 & 8 & 44.09 & -4.4 & 0.88 & 79.84 & 1.88 & 0.93 \\
\hline$\sigma_{M L}$ & 14 & 15.47 & -2.3 & 0.614 & 14.61 & 1.53 & 0.63 & 12 & 30.82 & -3.6 & 0.67 & 51.66 & 1.87 & 0.67 \\
\hline$\sigma_{I S}$ & 19 & 22.08 & -2.1 & 0.74 & 18.17 & 1.09 & 0.81 & 17 & 42.59 & -3.4 & 0.74 & 38.91 & 1.23 & 0.795 \\
\hline$\sigma_{a t !}$ & 41 & 24.15 & -4.03 & 0.74 & 19.9 & 1.48 & 0.68 & 37 & 44.55 & -5.0 & 0.72 & 50.95 & 1.60 & 0.66 \\
\hline
\end{tabular}

Table 3. Linear regression models for ultimate stress and modulus in compression tests to failure: $\sigma=a E+b$, where $\sigma=$ ultimate stress in units of $\mathrm{MPa}$, and $E=$ modulus in units of $\mathrm{MPa}$

\begin{tabular}{lrlll}
\hline & $n$ & $a$ & $b$ & $r^{2}$ \\
\hline$\sigma_{A P}$ & 35 & 0.028 & 1.23 & 0.80 \\
$\sigma_{M L}$ & 43 & 0.028 & 0.85 & 0.88 \\
$\sigma_{I S}$ & 52 & 0.031 & 0.14 & 0.76 \\
$\sigma_{\text {all }}$ & 130 & 0.03 & 0.61 & 0.83 \\
\hline
\end{tabular}

Comparison of relationships to human trabecular bone relationships

A complementary, larger scale study has been conducted at this laboratory on human trabecular bone from most of the major metaphyseal regions (Ciarelli et al., 1986). Since the orthogonal compression tests on human bone were performed using specimen geometry and test protocols identical to this study of canine bone, a comparison of the results seems a valid approach to establishing the limitations of canine trabecular bone as a model for human. Only the data from the human distal femur was used in the comparisons.

Qualitative similarities between canine and human trabecular bone could be found in their average mechanical and mass properties (Table 4). The large standard deviations in the modulus data for both species reflected the dependence of properties on metaphyseal location. It was noted that in both the canine and human distal femur, modulus values progressively decreased in the proximal direction. Though canine trabecular bone displayed lower moduli than human in all three directions, the patterns of mechanical anisotropy paralleled those demonstrated for the human distal femur, as the greatest stiffnesses were documented in the IS direction, followed by the AP, and finally the ML. It was also found that the average ash weight and apparent density of trabecular bone in the human and canine distal femur were not significantly different $(p>0.5)$.

Another method to establish the limitations of canine trabecular bone as a model for human was to compare the relationships between mechanical properties and mass properties. Before making such statistical comparisons, each mechanical and mass variable was adjusted for differences between animals and humans by subtracting the mean values for each animal and human from the raw data. Though it can be argued that independence is probably satisfied by the nature of Wolff's Law, this modification produced a much more conservative analysis and thus a stricter test of differences than typically performed on similar data. Therefore, while all graphs and empirical relationships of mechanical vs mass properties are presented in raw form, all analyses of covariance used to statistically test the differences in relationships between the two species were performed on the data in this normalized form.

In addition to the finding of similar average apparent and ash weight densities, perhaps more importantly, no difference was found in the human and canine relationships between these two variables $(p>0.99)$. The graph of the raw data in Fig. 2 demonstrates this quantitative similarity. Both linear regression models revealed that the ratio of the mineral to the composite of mineral, organic, water and volatile inorganic components of the trabecular tissue was about 0.55 .

Table 4. Quantitative similarities in the average mechanical and mass properties of the canine and human distal femoral trabecular bone

\begin{tabular}{llllll}
\hline & & \multicolumn{2}{c}{ Canine } & \multicolumn{2}{c}{ Human } \\
\hline AP modulus $(\mathrm{MPa})$ & 209 & $(140)$ & 298 & $(224)$ \\
ML modulus $(\mathrm{MPa})$ & 158 & $(110)$ & 203 & $(196)$ \\
IS modulus $(\mathrm{MPa})$ & 264 & $(132)$ & 424 & $(208)$ \\
Ultimate stress $(\mathrm{Mpa})$ & 7.12 & $(4.6)$ & 5.6 & $(3.8)$ \\
Apparent density $\left(\mathrm{g} \mathrm{cm}^{-3}\right)$ & 0.444 & $(0.16)$ & 0.430 & $(0.15)$ \\
Ash weight density $\left(\mathrm{g} \mathrm{cm}^{-3}\right)$ & 0.263 & $(0.08)$ & 0.255 & $(0.08)$ \\
\hline
\end{tabular}




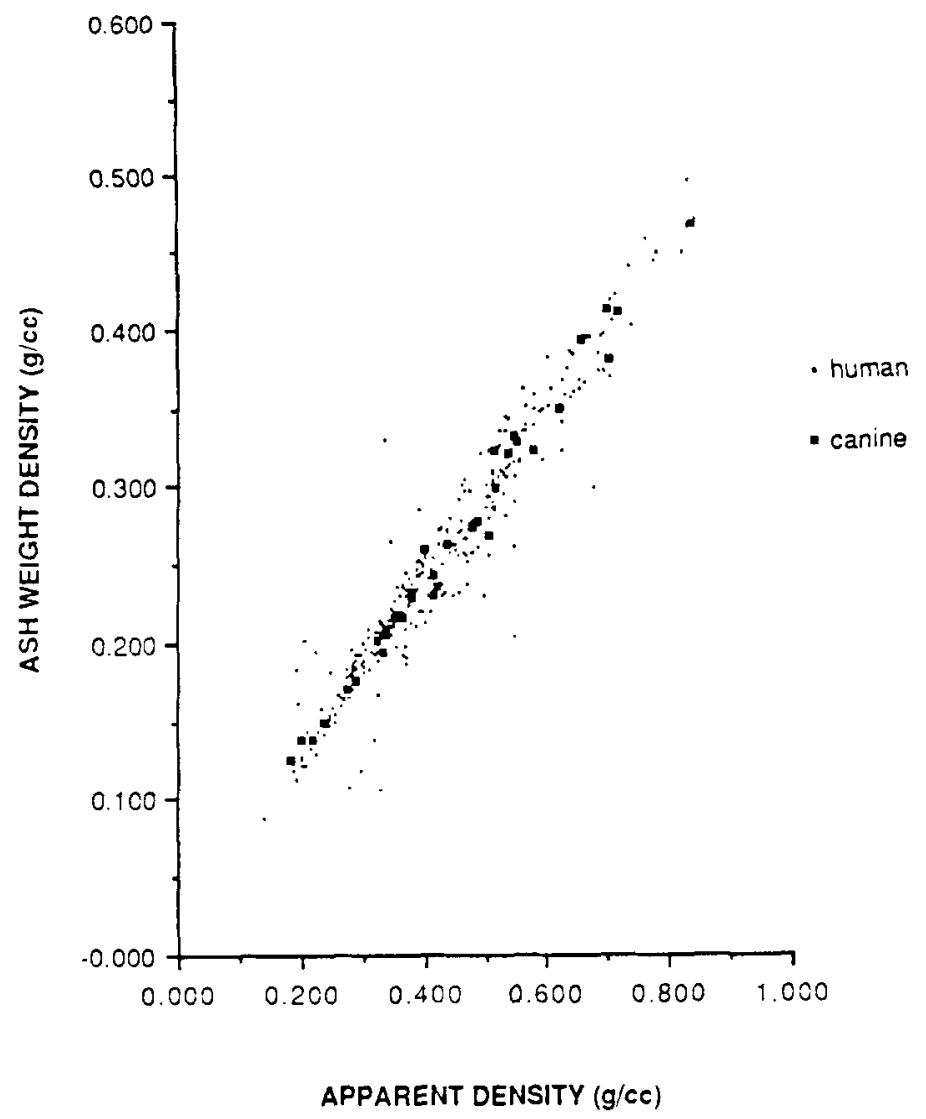

Fig. 2. No significant difference was found in the relationships between ash weight density and apparent density of canine and human trabecular bone.

Graphs of human and canine mean modulus versus apparent and ash weight density are shown in Fig. 3. Also shown are the best fit linear and power equations which represent the mean modulus as a function of density. Analyses of covariance were performed on the normalized data to test for the significance of the difference in these relationships. With respect to the linear regression models: with a $p=0.0019$, the slope of the human mean modulus-apparent density curve was significantly greater than the canine curve. For the analysis of the mean modulus-ash weight density curves, the difference was significant at a $p=0.0022$, also indicating the slope of the human curve to be greater than that for the canine. With respect to the $\log -\log$ regression models: significance $(p=0.033)$ was achieved in a test of the difference in intercepts of the log mean modulus-log apparent density curves, but no significant difference was found in the slopes nor intercepts of the log mean modulus-log ash weight density curves.

The empirical relationships derived from linear and $\log -\log$ regressions of the modulus in each test direction on apparent and ash weight density are shown in Table 5. Based on a comparison of the strength of the regressions, neither approach proved superior to the other. Both analyses showed that differences in density could explain approximately $33-68 \%$ of the variation in modulus data, depending on the direction of analysis. Also listed in Table 5 are $p$ values from analyses of covariance on the normalized data. In the majority of cases, significance was either achieved or approached in tests of differences between canine and human relationships. Only the $\log -\log$ relationships existing between each directional modulus and ash weight density demonstrated no significant difference between the two species.

Displayed in Fig. 4 are plots of ultimate stress vs apparent and ash weight density, and linear and power equations relating these variables. In an analysis of covariance of the human and canine linear relationships no significant difference was found $(p$ $=0.59$ for apparent density, $p=0.53$ for ash weight density). For the comparison of $\log -\log$ relationships between these two species, the same result was obtained, though borderline for the case of apparent density ( $p=0.07$ for apparent density, $p=0.19$ for ash weight density).

Figure 5 shows a plot of canine and human ultimate stress vs modulus, combining all test directions from compression tests to failure. These relationships were significantly different $(p<0.0001)$, with the canine bone demonstrating a higher slope. 


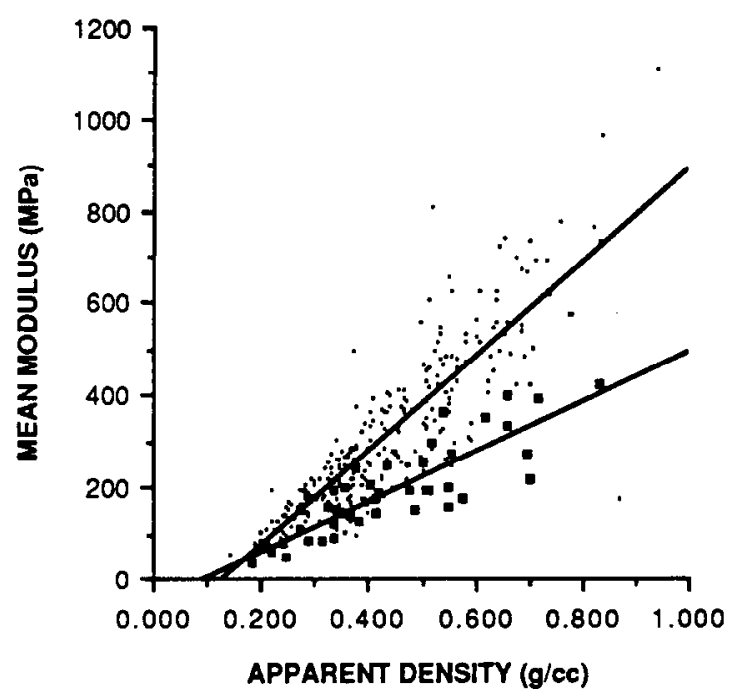

linear models

- canine: $y=-47.9+547.1 x \quad R=0.86$

- human: $y=-128.9+1024.3 x \quad R=0.87$

Dower models

- canine: $y=572.5 x^{1.39}$

- human: $y=992.3 x^{1.46}$

$R=0.89$



linear models

- canine: $y=-62.8+991.4 x \quad R=085$

- human: $y=-177.7+1920.2 x \quad R=088$

Dower models

- canine: $y=1352.9 x^{1.48} \quad R=088$

- human: $y=2416.3 x^{1.55} \quad R=088$

ASH WEIGHT DENSITY (g/cc)

Fig. 3. These graphs demonstrate the relationships between mean modulus and apparent and ash weight density for both canine and human trabecular bone of the distal femur. Best fit linear and power models are also shown.

\section{DISCUSSION}

A primary concern in orthogonal compression tests of trabecular bone is the ability to achieve-true preyield loading curves. It has been recently shown that the precision of the data is not only dependent on this ability to avoid permanent damage to the structure, but also relies on the preconditioning of bone specimens, as well as the percentage of ultimate stress at which a tangent modulus is calculated (Linde and Hvid, 1987). Thirty-five of the 130 specimens in this study were tested to preyield in three directions and then to failure in a randomly selected direction. Assuming this specimen subset to be a representative sample of the data, a level of precision in our modulus data was estimated.
Addressing first the problem of specimen failure in a presumed 'preyield' test, it could be seen that the probability of this occurrence was greater when relatively compliant trabecular bone specimens were tested. This observation can be better understood when one considers the somewhat ambiguous course of the load-deflection curve produced by low modulus specimens. Analyzing only those specimens with a modulus less than $170 \mathrm{MPa}$, the mean percent difference between the failure modulus and preyield modulus was $-19.9 \%$. Such a finding would be expected if damage had been incurred by the structure during the preyield tést. Unlike the low modulus specimens, stiffer specimens demonstrated clear indications of failure. Operator control over the loading of stiffer specimens was thus improved, with grcater assurance of a true 


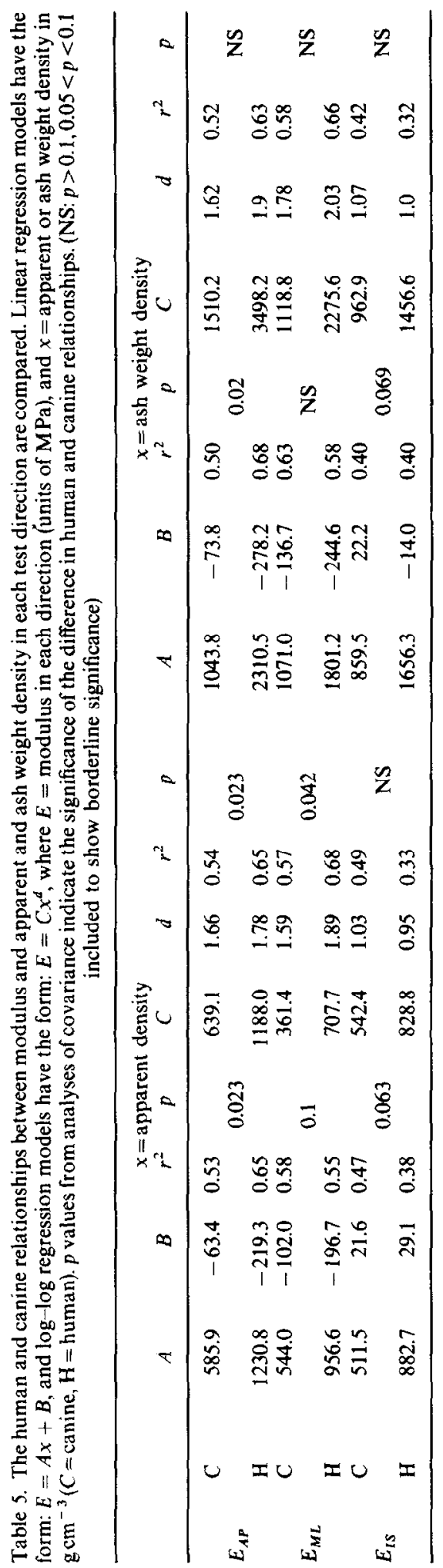

preyield condition. Consequently, a different trend was observed for stiffer specimens. In these cases, the preyield modulus tended to be about $3.6 \%$ less than the failure modulus.

Other errors might be introduced by measuring the tangent modulus at different points along the stress-strain curve. A specified percentage of the ultimate stress at which moduli are calculated is recommended by Linde and Hvid (1987) in order to avoid operator bias. In this study, the modulus values were in effect calculated from the most linear portion of the stress-strain curves rather than from a consistent percentage of ultimate stress. In the subset of 35 specimens, the linear regressions were performed predominantly around $45.2 \%$ of the ultimate stress with a range of $18-67 \%$.

Though general guidelines for mechanical testing are necessary and valuable, there is probably quite a range within the acceptable limits for testing parameters from one specimen to the next. The magnitude of the errors resulting from possible damage in a nondestructive compression test and the goals of the expcriment must be jointly considered in order to decide the level of required precision. We feel that the level of precision attained in our modulus measurements was adequate for investigating the variation in mechanical properties of canine trabecular bone, and the relationships between modulus, strength, and density.

Though the anatomic positions of the canine distal femur during gait are not identical to those of the human distal femur, some of the similarities in mechanical properties observed between the canine distal femur and human distal femur can be understood when the similarities in knee joint function are considered. First, the load bearing surfaces of the femur and tibia are located on the medial and lateral sides of the metaphysis, with the central region subjected to relatively low loads. As the metaphysis narrows and the diaphysis is approached, loads are transferred from the trabecular bone to the cortical shell which progressively increases in thickness. The positional variation of the mechanical properties within the distal canine femur reflect these differences in load carrying requirements. In accordance with Wolff's Law, the modulus and strength of the trabecular bone changed to meet the functional demands placed upon it.

The variation in mechanical anisotropy of the trabecular bone regions may also be explained by functional loads. For both the human and canine, the geometry of the femoral condyles and tibial plateau seem best suited for flexion-extension motion in the sagittal plane, allowing much less rotation in the coronal and transverse planes. As such, high anterior-posterior (AP with respect to the anatomy of an extended femur) forces as well as inferior-superior forces can be expected in regions of trabecular bone located near the articular surfaces. In regions proximal to the femoral condyles, body weight forces 

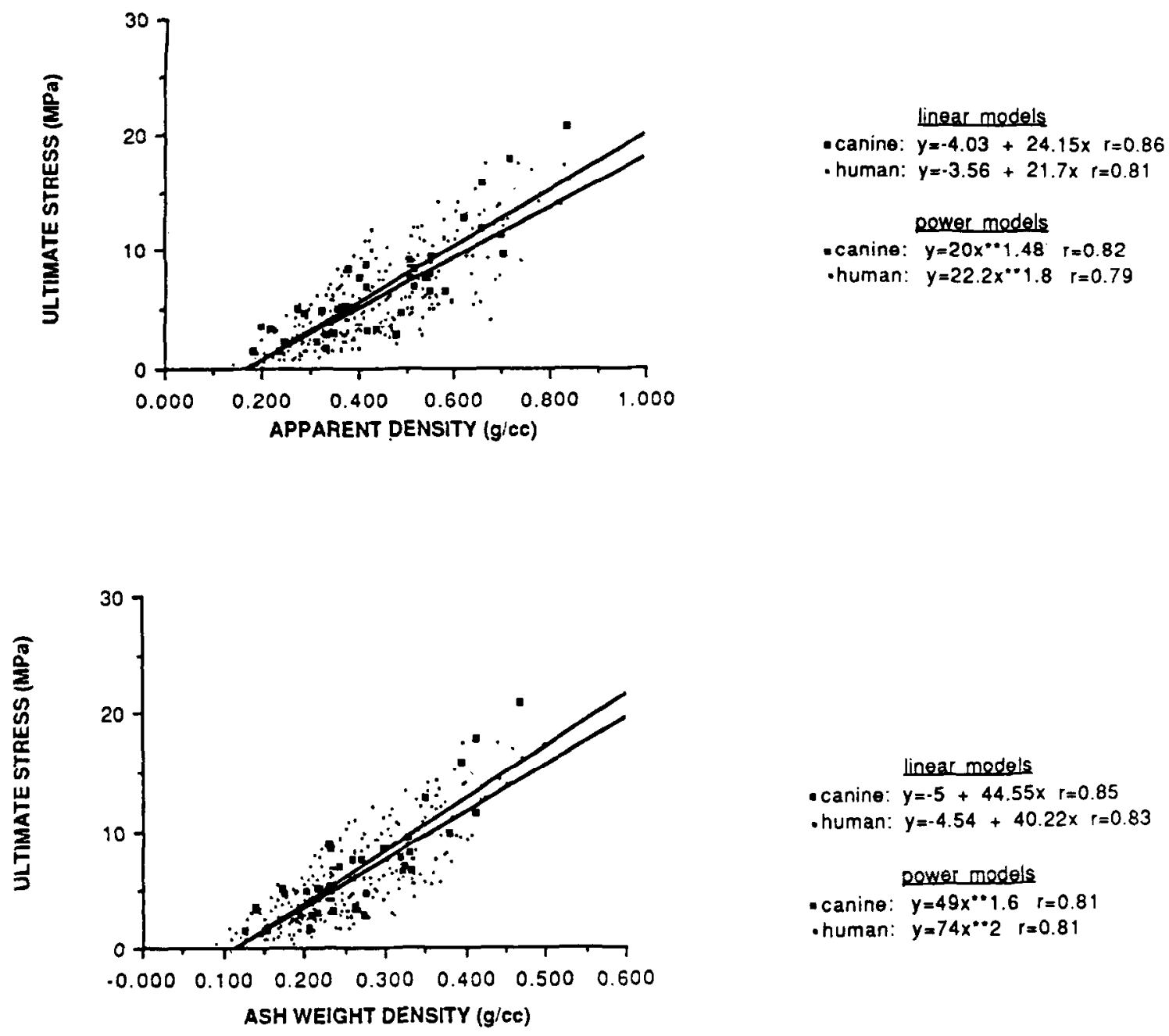

Fig. 4. These graphs of ultimate stress vs apparent and ash weight density for canine and human trabecular bone demonstrate no significant difference between these two species with respect to these relationships. While the best fit linear curves are displayed on the graphs, the equations of both the linear and power models are presented.

predominate in the IS direction with lower forces generated in the AP direction. Anterior regions of trabecular bone under the patellar groove also experience high compressive stresses. Our data demonstrated that the canine trabecular bone, like the human trabecular bone, possessed modulus and strength characteristics appropriate for the type of mechanical environment expected in these specific locations.

Interestingly, the ratio of the average IS and AP moduli for the canine distal femur was smaller than that for the human. This finding may reflect higher demands placed on the mechanical properties in the AP direction by the canine distal femur than by the human distal femur, as a result of its more flexed position during gait. Further and more detailed comparative investigations of the mechanical and structural anisotropy of the trabecular bone in these regions may illuminate the differences in kinematics and normal function of the knee joints in both species.
In support of the distal canine femur as a model for human, it was found that their average ash weight and apparent densities were not significantly different. More importantly, the relationships between ash weight density and apparent density in the canine and human were not significantly different. Although ash weight density and apparent density themselves are quantities normalized for bulk volume (tissue volume + pore volume), the ratio of these variables in a single specimen is a true measure of the fraction of components in the trabecular tissue of that specimen. Ash weight density is related to the mineral component while the apparent density is related to the mineral, organic, water, and volatile inorganic components in a specimen. Both the human data and the canine data showed that this ratio was about 0.54 to 0.55 . Gong $e t$ al. (1964) conducted a detailed experiment in which they compared the trabecular and cortical bone compositions of human, monkey, dog, and steer bones. Their data also showed that the fractions of the 



MODULUS (MPa)

Fig. 5. A significant difference in the canine and human relationships between ultimate stress and modulus can be seen in this graph.

constituents comprising the trabecular tissue were quantitatively similar between species. In addition, a ratio of ash weight density to apparent density can be calculated from their data and compared to the ratio found here. For their dog data, this ratio equalled 0.547 , and for their human data, 0.56 .

Another method to establish the limitations of canine trabecular bone as a model for human trabecular bone was to compare the relationships between mechanical properties and density. This analysis followed the lead of others who have used bone density as a first approach to explaining the variance in trabecular bone modulus and strength (Behrens and Walker, 1974; Carter and Hayes, 1977; Ciarelli et al., 1986; Ducheynne et al., 1977; Galante et al., 1970; Keller et al., 1986).

Both linear and power relationships proved to fit the data quite well. The strengths of the fits, as determined from a comparison of squared correlation coefficients, were very similar between the two types of analyses. In analyses by direction (AP, ML, IS), no clear evidence was found for a superior performance of either linear or power models. In analyses of combined direction data, power models explained $6 \%$ more of the variance in canine mean modulus than linear models, but linear models explained $6 \%$ more of the variance in canine ultimate stress than power models.
The power models for the canine data indicated a relationship of mean modulus to density raised to approximately 1.4-1.5, and for ultimate strength, density raised to approximately $1.5-1.6$. These results seem to contradict the results of Carter and Hayes (1977) which supported a density cubed relationship with modulus, and a density squared relationship with compressive strength. However, many different studies have analyzed trabecular bone from diverse anatomic regions and species and have reported quite a variation in the coefficients of these rclationships (Behrens and Walker, 1974; Carter and Hayes, 1977; Ciarelli et al., 1986; Ducheynne et al., 1977; Galante et al., 1970; Keller et al., 1986).

There may be several reasons for these observed discrepancies. First, all experiments have not used identical testing procedures, specimen shapes or dimensions. Presumably, all these results could be standardized to remove the effects of differing strain rate, by normalizing all data by a factor of strain rate raised to the 0.06 power, a relationship revealed by the study of Carter and Hayes (1977). However, the contributions of trabecular architecture and orientation are still unaccounted for by density measurements. As most previous studies have involved uniaxial compression or uniaxial strain tests along one chosen axis, the derived relationships may be dependent on the specific trabecular architecture in that direction. Also, one overlooked aspect which may be very relevant, is the range of bone densities represented by each sample population of bone specimens.

The study by Carter and Hayes (1977) incorporated data with a wide range of bone densities, and included bone specimens from different species. In fact these authors state that their empirically derived expressions. . may be used to estimate bone tissue compressive strength and modulus when considerable variations in the apparent density of bone exist', and that 'factors such as bone ultrastructure, mineralization, trabecular orientation (anisotropy), and disease state' should be considered when examining these same relationships 'over a narrow range of densities'. In their study, apparent densities seemed to range from approximately $0.1-2.0 \mathrm{~g} \mathrm{~cm}^{-3}$, while in this $s t u d y$, the range was approximately $0.2-0.8 \mathrm{~g} \mathrm{~cm}^{-3}$. Shown in Fig. 6 are curve plots of the expressions derived by Carter and Hayes for ultimate strength vs apparent density, and for compressive modulus vs apparent density, when a strain rate of $1 \% \mathrm{~s}^{-1}$ is used. Superimposed on these curves are the human and canine data of this study. When perceived on this grand scale, our data also seem to fall onto their curves with a comparable amount of scatter.

As the goals of these experiments were very different, the contradiction in the form of the relationships should not be a cause for alarm. The goal of this study was to compare trabecular bone from the same anatomical region of two different species, for which the range of apparent densities were small and similar. The tools of the comparison were statistical relation- 
$S=51.6 \times 2$ for strain rate $=1 \% \mathrm{sec}^{-1}$

(Carter and Hayes, 1977)
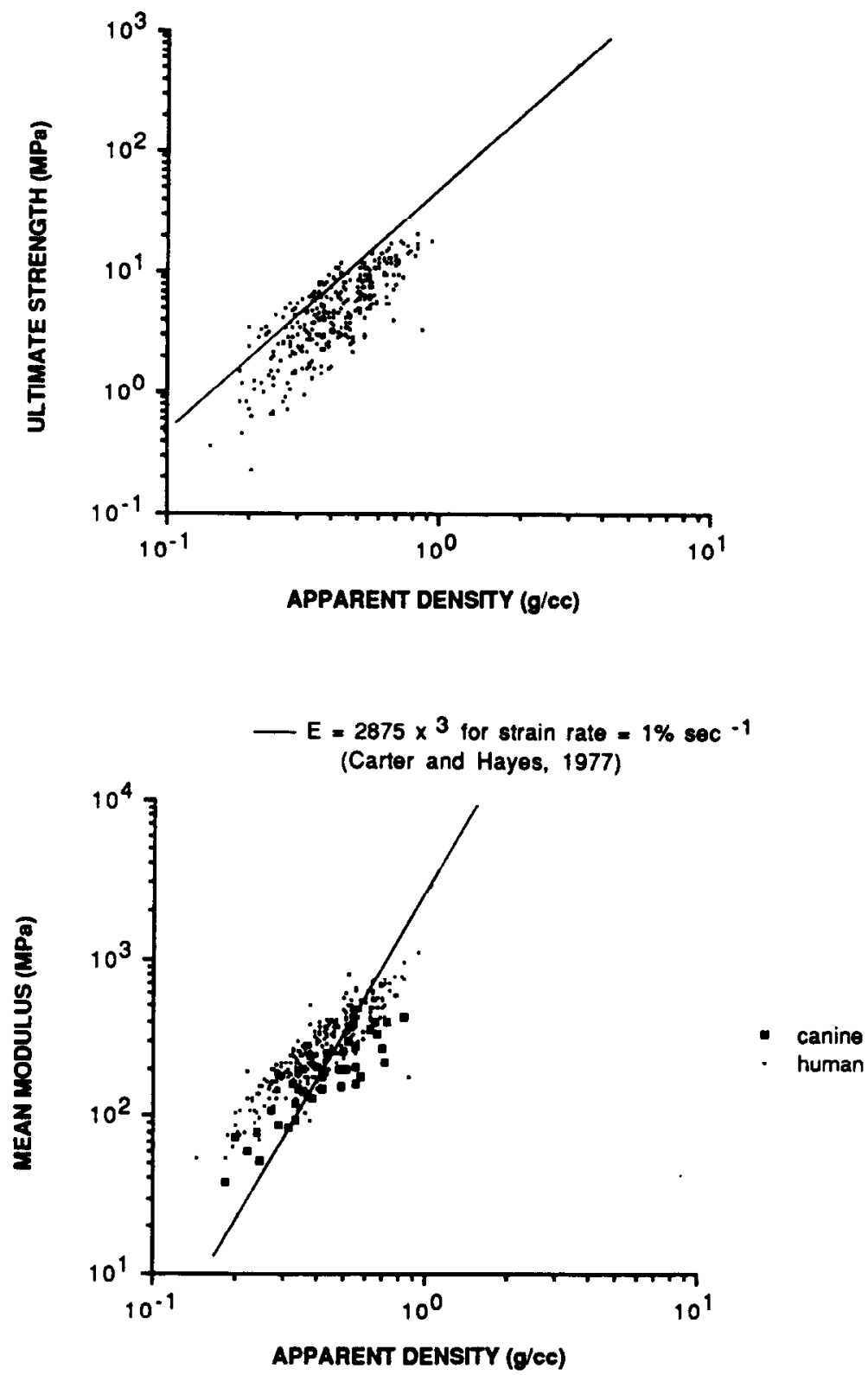

Fig. 6. Log-log plots of mean modulus and apparent density, and ultimate stress and apparent density are shown. Superimposed on these scatter plots of our data are curves representing similar expressions found by

Carter and Hayes (1977), when a strain rate of $1 \% \mathrm{~s}^{-1}$ is used.

ships and analyses of covariance. With this narrower range of densities, statistically significant differences were found which indicate potential differences in 'factors such as bone ultrastructure, mineralization, trabecular orientation (anisotropy), and disease state'. Lastly, the relationships presented in this paper are empirical relationships that describe the data as acquired under very specific conditions, and are not proposed as universal laws for canine or human trabecular bone.

The first indication of a difference between canine and human trabecular bone, came from a comparison of the canine and human relationships between mean modulus and apparent density. In both species, linear and $\log$ - $\log$ relationships were very strong. However, an analysis of covariance on the normalized data showed that after controlling for apparent density, the mean modulus of canine trabecular bone was less than that for human. Architectural factors may have played a role in this difference. Although the patterns of mechanical anisotropy in the trabecular bone of the canine and human distal femur were similar, the degree of anisotropy can be considerably different. The ratio between the maximum modulus and mini- 
mum modulus within each cube can be used as an index of the degree of anisotropy. This ratio averaged 3.7 for the human distal femur, and 2.6 for the canine distal femur, indicating that the human specimens possessed a greater degree of anisotropy than the canine specimens. This difference may have contributed to the difference in mean modulus-apparent density relationships. If, however, it is assumed that stratifying the data by anatomical directions reduces the variance in modulus due to architecture, then the comparison of canine and human relationships with apparent density shown in Table 5 tend to reaffirm the earlier result: that for the same apparent density, canine trabecular bone displays a lower mean modulus than human. The normalized mean modulus-ash weight density linear relationships also demonstrated a significant difference between the two species, supporting that for the same ash weight density the human mean modulus is higher than the canine. However, the statistical comparison of the normalized log mean modulus-log ash weight density human and canine regressions revealed no significant differences. The tendency for the ash weight density relationships to not be significantly different between species, may reflect the fact that the difference between ash weight density and apparent density is more than one of weight. The additional mass in the apparent density calculation also contributes further structural anisotropy in the form of collagen fiber orientations.

A comparison of ultimate compressive stress (all test directions) vs density relationships showed no significant difference between canine and human. These last two findings suggest that factors other than trabecular architecture, density, and tissue composition play a role. It may be plausible that architectural differences on a microstructural level exist. Equality in the fraction of tissue components does not necessarily imply equality in the organization of those components. Such a hypothesis could be tested by comparing the mechanical properties of canine and human trabecular tissue, and controlling for degree of mineralization, or by examining the collagen fibril orientations and the spatial arrangements of the bone mineral in the trabecular tissue of both species.

Finally, a statistical comparison of the normalized linear regression models of ultimate stress and modulus for canine and human demonstrated a significant difference between the trabecular bone of these two species. Actually, this result could have been predicted by the preceding results. In addition, since the data for this analysis included failure tests in all three directions (thereby including different architectural arrangements), further support is provided for a fundamental difference in the trabecular bone of the canine and human distal femur. If the regression coefficients of the linear models are considered to represent some measure of an ultimate strain limitation, then the data also implies that the trabecular bone of the canine distal femur reached a higher ultimate strain than human. Table 6 contains a partial summary from the literature of ultimate stress-modulus relationships derived from mechanical tests of both canine and human trabecular bone. Although there are large variations in the constants and coefficients of these equations, one common factor is that they are all linear. It can also be seen that the outcome of a comparison between canine and human relationships can be predetermined simply by a choice of which two papers to compare. The difference in equations could be due to differences in the types of bones tested, specimen geometries, or strain rates. We feel that the comparison made in this paper is not arbitrary, since identical experimental protocols were used to test the canine distal femurs and the human distal femurs.

Although many similarities exist between the mechanical properties and tissue properties of canine trabecular bone and human trabecular bone, this study seems to indicate that a difference may exist between

Table 6. Partial summary from the literature of compressive strength -modulus relationships for trabecular bone

\begin{tabular}{lll}
\hline Reference & Specimens and test parameters & \multicolumn{1}{c}{ Relationship } \\
\hline $\begin{array}{l}\text { Ducheynne } \text { et al. } \\
(1977)\end{array}$ & $\begin{array}{l}\text { Human femur, cylindrical } \\
\text { specimens, } d=5 \mathrm{~mm}\end{array}$ & $\begin{array}{l}S=2.16+.01 E(\mathrm{MPa}) \\
\text { strain rate }=2.08 \% \mathrm{~s}^{-1} \\
\mathrm{~S}=-2.2+0.03 E \\
\text { strain rate }=104 \% \mathrm{~s}^{-1}\end{array}$ \\
$\begin{array}{l}\text { Williams and } \\
\text { Lewis (1982) }\end{array}$ & $\begin{array}{l}\text { Human proximal tibia, 5-6mm } \\
\text { cubes, strain rate }=0.5 \% \mathrm{~s}^{-1}\end{array}$ & $S=0.487+.0137 E(\mathrm{MPa})$ \\
$\begin{array}{l}\text { Goldstein } \text { et al. } \\
(1983)\end{array}$ & $\begin{array}{l}\text { Human proximal tibia, } \\
\text { cylindrical specimens, } \\
\text { strain rate }=0.1 \% \mathrm{~s}^{-1}\end{array}$ & $S=0.0265 E(\mathrm{MPa})$ \\
$\begin{array}{l}\text { Brown and Ferguson } \\
(1980)\end{array}$ & $\begin{array}{l}\text { Human proximal femur, } \\
5 \mathrm{~mm} \text { cubes, strain } \\
\text { rate }=0.4 \% \mathrm{~s}^{-1}\end{array}$ & $S y=0.0416 E(\mathrm{MPa})$ \\
$\begin{array}{l}\text { Vahey } \text { et al. } \\
(1987)\end{array}$ & $\begin{array}{l}\text { Canine proximal femur, } \\
\text { rate }=0.1 \% \mathrm{~s}^{-1}\end{array}$ & $S=0.0288 E-0.422(\mathrm{MPa})$ \\
\hline
\end{tabular}


the two species, with respect to how the trabecular structure combined with its microstructure produces a continuum modulus.

A specimen size scaling effect could be raised as a possible source for the differences seen in this study. Eight $\mathrm{mm}$ bone cubes might seem large in relation to the size of a canine distal femur, and as such, a more variable architecture may be expected to exist within a volume of canine trabecular bone than within an equal volume of human trabecular bone from the same region. The optimal solution for avoiding this problem would be to pre-determine the appropriate boundaries for a specimen. This can be accomplished if one has available a means to noninvasively examine the entire structure of a bone volume before any physical cuts are made. The variation in architecture within $8 \mathrm{~mm}$ canine trabecular bone cubes from the distal femur has been investigated in another study (Kuhn, 1987). It was found that the canine bone exhibited significant structural variation, which may be indicative of a size scaling effect, in $13 \%$ of the samples, compared to $6 \%$ in the human samples. However, in light of this small percentage, we do not believe that the specimen size of the canine bone cubes was a strongly contributing factor to the differences seen between the trabecular bone properties of the canine and human distal femur in this study.

A limitation of this study was the inability to separate out the specific effects of individual architectural variables such as trabecular connectivity, orientation, and percent orientation on the displayed mechanical properties. Once such a generalized structure-function relationship is derived for trabecular bone, a more complete test of the difference between canine and human trabecular bone mechanical properties can be conducted.

\section{CONCLUSIONS}

Based on the findings of this study, the canine trabecular bane from the distal femur is qualitatively similar to human bone of the same region. The positional and anisotropic variation of the mechanical properties paralled established variations found in the human distal femur. As in human trabecular bone, apparent and ash weight density played an important role in determining the continuum stiffness and strength of canine bone. Quantitative similarities could be found in the percentage of components comprising the trabecular tissue of both species. However, it was found that quantitative differences existed in the relationships between mean modulus and density, and between ultimate stress and modulus. The difference in coefficients relating ultimate stress to modulus seemed to suggest that canine trabecular bone reached higher compressive strains before failure than human bone. Some unique characteristic of the canine trabecular bone microstructure may account for this difference, but at this point this is only pure speculation.
Acknowledgements-The authors wish to thank Dave Hak, Scott Hollister, and Ed Stokel for their assistance in the study. Support was provided by grants from the National Institutes of Health (AR 31793, AR 34399).

\section{REFERENCES}

Arnold, J. S. (1960) Quantitation of mineralization of bone as an organ and tissue in osteoporosis. Clin. Orthop. 17, $167-175$.

Behrens, J. C. and Walker, P. S. (1974) Variations in strength and structure of cancellous bone at the knee. J. Biomechanics. 7, 201-207.

Bobyn, J. D., Pilliar, R. M., Cameron, H. U. and Weatherly, G. C. (1980) The optimum pore size for the fixation of porous surfaced metal implants by the ingrowth of bone. Clin. Orthop Rel. Res. 150, 263-270.

Bos, G. D., Goldberg, V. M., Powell, A. E., Heiple, K. G. and Zika, J. M. (1983) The effect of histocompatibility matching on canine frozen bone allografts. J. Bone Jt. Surg. 65A, 89-96.

Brown, T. D. and Ferguson, A. B. (1980) Mechanical property distributions in the cancellous bone of the human proximal femur. Acta. scand. orthop. 51, 429-437.

Burr, D. B., Martin, R. B., Schaffler, M. B. and Radin, E. L. (1985) Bone remodeling in response to in vivo fatigue microdamage. J. Biomechanics 18, 189-200.

Carter, D. R. and Hayes, W. C. (1977) The compressive behavior of bone as a two-phase porous structure. J. Bone Jt. Surg. 59A, 954-962.

Chamay, A. and Tschantz, P. (1972) Mechanical influences in bone remodelling: experimental research on Wolffs Law. $J$. Biomechanics. 5, 173-180.

Ciarelli, M. I., Goldstein, S. A., Dickie, D., Ku, J. L., Kapper, M., Stanley, J., Flynn, M. J. and Matthews, L. S. (1986) Experimental determination of the orthogonal mechanical properties, density, and distribution of human trabecular bone from the major metaphyseal regions utilizing materials testing and computed tomography. Transactions of the 32nd Annual Meeting of the Orthopaedic Research Society. Vol. 11, p. 42.

Detenbeck, L. C. and Jowsey, J. (1969) Normal aging in bone of adult dog. Clin. Orthop. Rel. Res. 65, 76-80.

Ducheynne, P., Heymans, L., Martens, M., Aernoudt, E., De Meester, P. and Mulier, J. C. (1977) The mechanical behavior of intracondylar cancellous bone of the femur at different loading rates. $J$. Biomechanics 10, 747-762.

Enneking, W. F., Burchardt, H., Puhl, J. J. and Piotrowski, G. (1975) Physical and biological aspects of repair in dog cortical bone transplants. J Bone Jt Surg. 57A, 237-252.

Faugere, M. C., Rush, M., Friedler, R. M. and Malluche, H. H. (1985) Loss of ovarian function causes bone loss due to an osteoblastic deficiency in dogs. Clin. Research 33, 849A.

Galante, J., Rostoker, W. and Ray, R. D. (1970) Physical properties of trabecular bone. Calc. Tiss. Res. 5, 236-246.

Galante, J., Rostoker, W., Lueck, R. and Ray, R. D. (1971) Sintered fiber metal composites as a basis for attachment of implants to bone. J. Bone Jt Surg. 53A, 101-114.

Goldstein, S. A., Wilson, D. L., Sonstegard, D. A. and Matthews, L. S. (1983) The mechanical properties of human tibial trabecular bone as a function of metaphyseak location. J. Biomechanics. 16, 965-969.

Goldstein, S. A., Ku, J. L., Hollister, S. J., Kayner, D. C. and Matthews, L. S. (1986) The effects of applied stress on experimentally controlled remodeling in trabecular bone. Transactions of the 32nd Annual Meeting of the Orthopaedic Research Society, Vol. 11, p. 432.

Gong, J. R., Arnold, J. S. and Cohn, S. H. (1964) Composition of trabecular and cortical bone. Anat. Rec. 149, 325-331.

Harris, W. H., Jackson, R. H. and Jowsey, J. (1962) The in vivo distribution of tetracyclines in canine bone. $J$. Bone $J t$ Surg. 44A, 1308-1320. 
Harris, W. H., Heaney, R. P., Jowsey, J., Cockin, J., Akins, C., Graham, J. and Weinberg, E. H. (1972) Growth hormone: the effect on skeletal renewal in the adult dog, I. Morphometric studies. Calc. Tiss. Res., 10, 1-13.

Jee, W. S. S. (1980) Age related biology of the skeleton of carnivores and man. The Use of Carnivores as Models for Research in Aging. Institute of Laboratory Animal Resources, Division of Biological Sciences, National Research Council, Assembly of Life Sciences, Washington, DC.

Keller, T. S., Hansson, T. H., Panjabi, M. M. and Spengler, D. M. (1986) Regional variations in the compressive properties of human lumbar vertebral trabeculae: influence of tissue physical characteristics. Proceedings of the North American Congress on Biomechanics, Vol. 1, p. 147.

Kuhn, J. L. (1987) Trabecular bone structure and mechanical properties. Ph.D. Dissertation, University of Michigan, Ann Arbor.
Linde, F. and Hvid, I. (1987) Stiffness behavior of trabecular bone specimens. J. Biomechanics 20, 83-90.

Martin, R. B., Albright, J. P., Jee, W. S. S., Taylor, G. N. and Clarke, W. R. (1981) Bone loss in the beagle tibia: influence of age, weight, and sex. Calcif. Tissue Int. 33, 233-238.

Snow, G. R. and Anderson, C. (1986) The effects of 17 estradiol and progestagen on trabecular bone remodeling in oophorectomized dogs. Calcif Tissue Int. 39, 198-205.

Snow, G. R., Cook, M. A. and Anderson, C. (1984) Oophorectomy and cortical bone remodeling in the beagle. Calcif Tissue Int. 36, 586-590.

Vahey, J. W., Lewis, J. L. and Vanderby, R. Jr (1987) Elastic moduli, yield stress, and ultimate stress of cancellous bone in the canine proximal femur. J. Biomechanics 20, 29-34.

Williams, J. L. and Lewis, J. L. (1982) Properties and an anisotropic model of cancellous bone from the proximal tibial epiphysis. J. Biomed. Engng. 104, 50-56. 